\title{
Quintessence and the first Doppler peak
}

\author{
Pedro F. González-Díaz ${ }^{1}$ \\ Instituto de Matemáticas y Física Fundamental, Consejo Superior de \\ Investigaciones Científicas, Serrano 121, 28006 Madrid, Spain
}

\begin{abstract}
By using a tracking quintessence model we obtain that the position of the first Doppler peak in the spectrum of CMB anisotropies only depends on the topology of the universe, $\Omega_{k}$, for any value of the ratio $\Omega_{\Lambda} / \Omega_{M}$, so that such a dependence is perfectly valid in the range suggested by supernova observations.
\end{abstract}

Subject headings: cosmology: field-theory models

\section{Introduction}

Recent experiments and observations have already opened up what one can actually call the era of precision cosmology. It is now hoped that next years will see rather impressive advances leading not just to the determination of key cosmological parameters with an accuracy not even dreamed five years ago, but also to unprecedented scrutinies on fundamental aspects of particle and field theories. In particular, the connection between recent Boomerang (de Bernardis et al. 2000) and Maxima (Hanany et al. 2000) experiments with theory is twofold. They are linked through predictions from either inflationary (and perhaps cosmic string) models by using fundamental particle physics arguments ( $\mathrm{Hu}$ and White 1996) or from general more or less standard cosmological scenarios which may or may not include some new concepts such as quintessence (Cadwell, Dave and Steinhardt 1998). The aim of this paper will concentrate at a particular aspect of CMB anisotropy measurements: the dependence of the position of the first Doppler peak on the values of the relevant cosmological parameters within the realm of a quintessence model. Kamionkowski, Spergel and Sugiyama (1994) originally derived the simple relation that the position of the first Doppler peak $\ell \sim 200 \Omega_{0}^{-1 / 2}$, where $\Omega_{0}=\Omega_{M}+\Omega_{\Lambda}$, which has been the subject of some controversy. While Frampton, Ng and Rohm (1998) have recently derived a similar dependence using a simple quintessential model, Weinberg (2000) has argued that this formula is not

\footnotetext{
${ }^{1}$ Centro de Física ”M.A. Catalán”, E-mail: p.gonzalezdiaz@imaff.cfmac.csic.es
} 
even a crude approximation when $\Omega_{M}$ is smaller than $\Omega_{\Lambda}$. For a very recent discussion on quntessential model of CMB anisotropies see Bond et al. (2000). Assuming the holding of the cosmic triangle condition, we obtain in this paper that $\ell$ only depends on the topology of the universe, so confirming the original proposal by Kamionkowski, Spergel and Sugiyama (1994).

\section{Calculation of $\ell$}

In order to derive an expression relating the position of the first Doppler peak with the scalar field potential of quintessence models, we choose a general tracking model with time-dependent parameter $\omega(t)$ for the state equation (Zlatev, Wang and Steinhardt 1999). This kind of models can be related to particle physics and may solve the so-called cosmic coincidence problem (Steinhardt 1997). One typically considers tracking quintessence fields $\phi$ for a Ratra-Peebles potential $V(\phi) \propto \phi^{-\alpha}$ with a given constant parameter $\alpha$ (Ratra and Peebles 1988). During its cosmic evolution, the equation of state parameter $\omega$ passes through several distinct regimes (Brax, Martin and Riazuelo 2000), including first a kinetic regime lasting until $z \sim 10^{20}$ with $\omega=+1$, then the transition and potential regimes chracterized by $\omega=-1$, to finally reach the proper tracking regime at $z \sim 10^{15}$, where $\omega=\left(\alpha \omega_{B}-2\right) /(\alpha+2)$ (with $\omega_{B}=1 / 3$ for radiation dominated universe and $\omega_{B}=0$ for matter dominated universe). On this regime, there exists a particular solution for the scalar field, $\phi=\phi_{0} \eta^{4 /(\alpha+2)}$ (in which

$\eta=\int d t / R(t)$ is the conformal time, with $R(t)$ the scale factor) which is an attractor able to solve the cosmic coincidence problem.

For $\alpha \neq 0$ a change of $\omega$ should then be expected when the universe becomes matter dominated once the the surface of last scattering is overcome. Since the tracking regime is characterized by $\omega=c_{s}^{2}\left(c_{s}^{2}\right.$ being the sound velocity defined as $\left(d p_{\phi} / d \eta\right) /\left(d \rho_{\phi} / d \eta\right)(\operatorname{Brax}$, Martin and Riazuelo 2000)), parameter $\omega$ becomes a constant given by $\omega=-2 /(\alpha+2)$ when matter dominates. It has been recently argued that $\alpha \leq 2$ (Balbi et al. 2001), so that if we choose for definiteness $\alpha=1$, then $\omega=-2 / 3$ for the vacuum quintessence field. In this case, the Ratra-Peebles potential becomes

$$
V(\phi) \propto \phi^{-1}
$$

Since the corresponding tracking solution, $\phi \propto \eta^{4 / 3}$, would then correspond to a constant parameter $\omega<0$ for the quintessence field, it should satisfy the constraint equation derived from the corresponding conservation laws and cosmological field equations (Di Pietro and Demaret 1999, González-Díaz 2000). In terms of the cosmological parameters $\Omega_{i}, i=M, \phi, \Lambda, k$, and the quintessence field potential $V(\phi)$, this constraint equation can be written as (González- 
Díaz 2000)

$$
\begin{gathered}
\left(\frac{V^{\prime}}{V_{0}^{\prime}}\right)^{2}= \\
\Omega_{M}\left(\frac{V}{V_{0}}\right)^{(\omega+2) /(\omega+1)}+\Omega_{\phi}\left(\frac{V}{V_{0}}\right)^{2}+\Omega_{k}\left(\frac{V}{V_{0}}\right)^{(3 \omega+1) /[3(\omega+1)]}+\Omega_{\Lambda}\left(\frac{V}{V_{0}}\right)^{(3 \omega+4) /[3(\omega+1)]},
\end{gathered}
$$

where $^{\prime} \equiv d / d \phi$, the subscript 0 means current value and $V_{0} / v_{0}^{\prime}= \pm \sqrt{3 \Omega_{\phi} /(1+\omega)}$. Eqn. (2) corresponds to the generalized quintessence model with negative constant parameter for the state equation recently suggested (González-Díaz 2000). Besides the contributions from the topological curvature, $k$, and gravitationally observable mass, $M$, this model distinguishes two essentially distinct contributions from vacuum energy: a varying cosmological term with positive energy density $\Omega_{\Lambda}$ assumed to satisfy the conservation law $8 \pi G \rho_{\Lambda}=\Lambda_{0}\left(R_{0} / R\right)$ at sufficiently small redshifts, and a quintessence negative energy density such that while $\Omega_{\phi}=8 \pi G \rho_{\phi} / 3 H_{0}^{2}$ ( $H_{0}$ being the current value of the Hubble constant) is always negative, $\Omega_{v} \equiv \Omega_{\Lambda}+\Omega_{\phi}$ is always positive, so satisfying the Ford-Roman's quantum interest conjecture (Ford and Roman 1999).

Adapting then the relation between the position of the first Doppler peak $\ell$ and the cosmological parameters $\Omega_{i}$ first derived by Frampton, Ng and Rohm (1998)to our generalized model, we can finally obtain a general relation between $\ell$ and the quintessence potential $V(\phi)$ of the form:

$$
\begin{gathered}
\ell=\frac{6 \pi(1+\omega) V_{0}}{(3 \omega+1) \sqrt{\left|\Omega_{k}\right|} V_{0}^{\prime}} \times \\
\left.\frac{d}{d \phi}\left[\left(\frac{V}{V_{0}}\right)^{(3 \omega+1) /[6(\omega+1)]}\right]\right|_{z=Z_{R}} S\left\{\frac{\sqrt{\left|\Omega_{k}\right|} V_{0}^{\prime}}{3(\omega+1) v_{0}} \int_{\phi(z=0)}^{\phi\left(z=z_{R}\right)} \frac{d \phi}{\left(\frac{V}{V_{0}}\right)^{(3 \omega+1) /[6(\omega+1)]}}\right\},
\end{gathered}
$$

where $z_{R}=1100$ is the redshift at recombination, we have used the relation (Di Pietro and Demaret 1999)

$$
\frac{V}{V_{0}}=\left(\frac{R_{0}}{R}\right)^{3(\omega+1)}=(1+z)^{3(\omega+1)},
$$

and $S(x)=1$ for $\Omega_{k}=0, S(x)=\sin (x)$ for $\Omega_{k}<0$ and $S(x)=\sinh (x)$ for $\Omega_{k}>0$. In the case of interest $\omega=-2 / 3$, where we obtain both topological and dynamical acceleration, Eqn. (3) reduces to

$$
\ell=\left.\frac{2 \pi V_{0}}{\sqrt{\left|\Omega_{k}\right|} V_{0}^{\prime}} \frac{d}{d \phi}\left[\left(\frac{V}{V_{0}}\right)^{-1 / 2}\right]\right|_{z=z_{R}} S\left\{\frac{\sqrt{\left|\Omega_{k}\right|} V_{0}^{\prime}}{V_{0}} \int_{\phi(z=0)}^{\phi\left(z=z_{R}\right)} d \phi\left(\frac{V}{V_{0}}\right)^{1 / 2}\right\}
$$

and there is a solution to the constraint equation (2) given by

$$
V=V_{0}\left\{\sinh \left[\beta_{0}\left(\phi-\phi_{0}\right)\right]+\xi_{0}\right\}^{-1},
$$


where

$$
\begin{gathered}
\beta_{0}= \pm \frac{1}{3} \sqrt{\frac{\Omega_{\Lambda}+\Omega_{\phi}}{\Omega_{\phi}}}= \pm \frac{1}{3} \sqrt{\frac{\Omega_{v}}{\Omega_{\phi}}} \\
\xi_{0}=-\frac{\Omega_{k}}{2 \Omega_{v}} .
\end{gathered}
$$

In this case, the cosmological parameters must satisfy the relations

$$
\begin{gathered}
\Omega_{v}=\frac{1}{4}\left[1-\Omega_{k}+\sqrt{1-2 \Omega_{k}-\Omega_{k}^{2}}\right] \geq 0 \\
\Omega_{M}=\frac{1}{4}\left[3\left(1-\Omega_{k}\right)-\sqrt{1-2 \Omega_{k}-\Omega_{k}^{2}}\right]>0 .
\end{gathered}
$$

Together with the triangle equation $\Omega_{v}+\Omega_{M}+\Omega_{k}=1$, Eqns. (9) and (10) will restrict the values that the cosmological parameters may take on. On the other hand, we note from Eqns. (4) and (6) that for large $z$ and $\Omega_{k} \sim 0$ one obtains $\phi \propto \eta^{2} \propto R$ and $V \propto \phi^{-1} \propto R^{-1}$; that is, while the potential (6) can be consistently interpreted as a generalization for smaller values of $z$ from the Ratra-Peebles potential, the attractor solution $\phi \propto R^{4 / 3}$ must necessarily change into $\phi \propto R$ as the universe becomes matter dominated.

Performing the derivative and integration in Eqn. (5) after inserting solution (6), we finally obtain for the position of the first Doppler peak when $\omega=-2 / 3$

$$
\ell=\pi \sqrt{\frac{\Omega_{v}\left(1+z_{R}\right)}{\left|\Omega_{k}\right|}\left[1+\left(\frac{1}{1+z_{R}}-\frac{\left|\Omega_{k}\right|}{2 \Omega_{v}}\right)^{2}\right]} T\left\{\left.\sqrt{\frac{\left|\Omega_{k}\right|}{\Omega_{v} \varphi_{0}}} F\left[\varphi(z), r_{0}\right]\right|_{0} ^{z_{R}}\right\}
$$

where $F\left[\varphi, r_{0}\right]$ is the elliptic integral of the first kind (Abramowitz and Stegun 1965),

$$
\begin{gathered}
\varphi=\arccos \left(\frac{\varphi_{0}-\frac{1}{1+z}}{\varphi_{0}+\frac{1}{1+z}}\right), \\
r_{0}=\left(\frac{\frac{\left|\Omega_{k}\right|}{2 \Omega_{v}}+\varphi_{0}}{2 \varphi_{0}}\right)^{1 / 2} \\
\varphi_{0}=\sqrt{1+\left(\frac{\left|\Omega_{k}\right|}{2 \Omega_{v}}\right)^{2}}
\end{gathered}
$$

and $T(x)=1$ for $\Omega_{k}=0$ and, unlike the function $S(x), T(x)=\sin (x)$ both for the closed and open cases. $\Omega_{k}$ can run between two extreme values, such that

$$
-(1+\sqrt{2}) \leq \Omega_{k} \leq \sqrt{2}-1 .
$$


A plot for the position of the first Doppler peak, $\ell$, against the parameter $\Omega_{k}$ is given in Fig. 1. It can be seen that the maximum value for $\ell$ is reached when $\ell$ approaches the value 190 at the flat case $\Omega_{k}=0$, decreasing slowly therefrom as $\left|\Omega_{k}\right|$ increases, a little more steeply for $\Omega_{k}>0$ than for $\Omega_{k}<0$. This seems to quite reasonably reproduce the results provided by Boomerang, Maxima and previous (Netterfield et al. 1997, Vollaek et al. 1997) experiments. Although uncertainties about our results for $\omega=-2 / 3$ must come from the possibility of having values of $\alpha$ other than just unity (provided they are on the interval $(0,2)$, or small deviation from time-independence of $\omega$ during cosmic evolution after recombination, they seems to strongly suggest a nearly flat topology for the universe.

We note that CBM anisotropies and cosmic acceleration can be easily related in our model. To see this, let us consider solution (6) for the the flat case, i.e.

$$
V=V_{0}\left\{\sinh \left[ \pm \sqrt{\frac{\Omega_{M}}{\Omega_{\phi}}}\left(\phi-\phi_{0}\right)\right]\right\}^{-1}
$$

with

$$
\Omega_{v}=\Omega_{M}=\frac{1}{2}
$$

This allows us to also construct a plot for the luminosity distance versus redshift by using the expression (González-Díaz 2000)

$$
D_{L} H_{0}=\sqrt{2}(1+z)\left\{\left.F\left[\arccos \left(\frac{1-\frac{1}{1+z^{\prime}}}{1+\frac{1}{1+z^{\prime}}}\right), 45^{\circ}\right]\right|_{0} ^{z}\right\}
$$

One can see readily that our flat solution in fact gives rise to a $5 \log D_{L} H_{0}-z$ plot with a nearly straight line between $z \simeq 0.01$ and $z \simeq 0.5$ which appears to slightly accelerate thereafter, the full $5 \log D_{L} H_{0}$ that corresponds to the $z$-interval of presently available type Ia supernova observations, $[\simeq(0.01-1)]$, being around 12 . This accelerating behaviour for the universe conforms quite well the data obtained from distant supernova Ia (Perlmutter et al. 1999, Reiss et al. 1998). It is worth noticing that though our universe model is dynamically accelerating it still is topologically uniform as $q_{0}=\frac{1}{2}\left(\Omega_{M}-\Omega_{v}\right)$ exactly vanishes in the flat case. As one separates from flatness one model produces topologically decelerating scenarios.

\section{Conclusions}

Consistent solutions to the constraint equation for the quintessence potential have been obtained which all correspond to particular fixed sets of values for $\Omega_{i}, i=k, M, \phi, \Lambda$, in such a 
way that the resulting value of the position of the first Doppler peak $\ell$ becomes automatically fixed once just one of the parameters $\Omega_{k}, \Omega_{M}$ or $\Omega_{v}$ is fixed, but does not depend on the ratio $\Omega_{\phi} / \Omega_{\Lambda}<0$. One can also conclude that $\ell$ acquires a maximum value which fits fairly well experimental results at $\Omega_{k}=0$ (i.e. $\Omega_{M}=\Omega_{v}=1 / 2$ ). As the topology of the universe separates from flatness $\left(\Omega_{M}, \Omega_{v}>1 / 2\right.$ if $\Omega_{k}<0$, or $\Omega_{M}, \Omega_{v}<1 / 2$ if $\left.\Omega_{k}>0\right)$, $\ell$ always decreases from its maximum value. Since $\Omega_{\phi}<0$, from the condition that $V / V_{0}$ be real it follows that the quintessence field $\phi$ is pure imaginary. This amounts to the interpretation that the classical solution is axionic, and hence the quintessence field can be taken as a genuine component of dark matter in such a way that one can define for the gravitationally observable mass of the universe the quantity $\Omega_{M}^{\prime}=\Omega_{M}+\Omega_{\phi}>0$, with $\Omega_{v}>0$. Thus, one can always adjust our results to the currently favoured combination $\Omega_{M}^{\prime}=0.3, \Omega=0.7$ for any $\Omega_{k}$.

We finally remark that the results obtained in this paper refer only to the case of a quintessence parameter for state equation $\omega=-2 / 3$ (i.e. $\alpha=1$ ). It is expected that results even more adjusted to observation (i.e. closer to 200) can also be obtained within the model of this paper by slightly shifting $\omega$ towards more realistic smaller values approaching -0.8 .

For helpful comments, the author thanks C. Sigüenza. This research was supported by DGICYT under research project No. PB97-1218.

\section{REFERENCES}

Abramowitz, M., and Stegun, I.A., 1965, Handbook of Mathematical Functions: Dover, 1995

Balbi, A., Baccigalupi, C., Matarrese, S., Perrotta, F., and Vittorio, N. 2001, ApJ, 547, L89

de Bernardis, P. et al. 2000, Nature, 404, 955

Bond, J.R. et al. 2000, astro-ph/0011379

Brax, P., Martin, J., and Riazuelo, A. 2000, Phys. Rev., D62, 103505

Caldwell, R.R., Dave, R., and Steinhardt, P.J. 1998, Phys. Rev. Lett. 80, 1582

Di Pietro, E., and Demaret, J. 1999, gr-qc/9908071

Ford, L.H., and Roman, T.A. 1999, Phys. Rev. D60, 104018

Frampton, P.H., Ng, Y.J., Rohm, R.M. 1998, Mod. Phys. Lett. A13, 2541 
González-Díaz, P.F. 2000, Phys. Rev. D62, 023513

Hanany, S. et al. 2000, ApJ, 545, 1

Hu, W., and White, M. 1996, ApJ, 471, 30

Kamionkowski, K, Spergel, D.N., and Sugiyama, N. 1994, ApJ, 426, 57

Netterfield, C.B. et al. 1997, ApJ, 474, 47

Perlmutter, S. et al. 1999, ApJ, 517, 565

Ratra, B., and Peebles, P.J.E. 1988, Phys. Rev. D37, 3406

Steinhardt, P.J. 1997, Critical Problems in Physics, V.L. Fitch and D.R. Marlow, Princeton: Princeton University Press

Weinberg, S. 2000, Phys. Rev. D62, 127302

Zlatev, L., Wang, L., and Steinhardt, P.J. 1999, Phys. Rev. Lett. 82, 896 


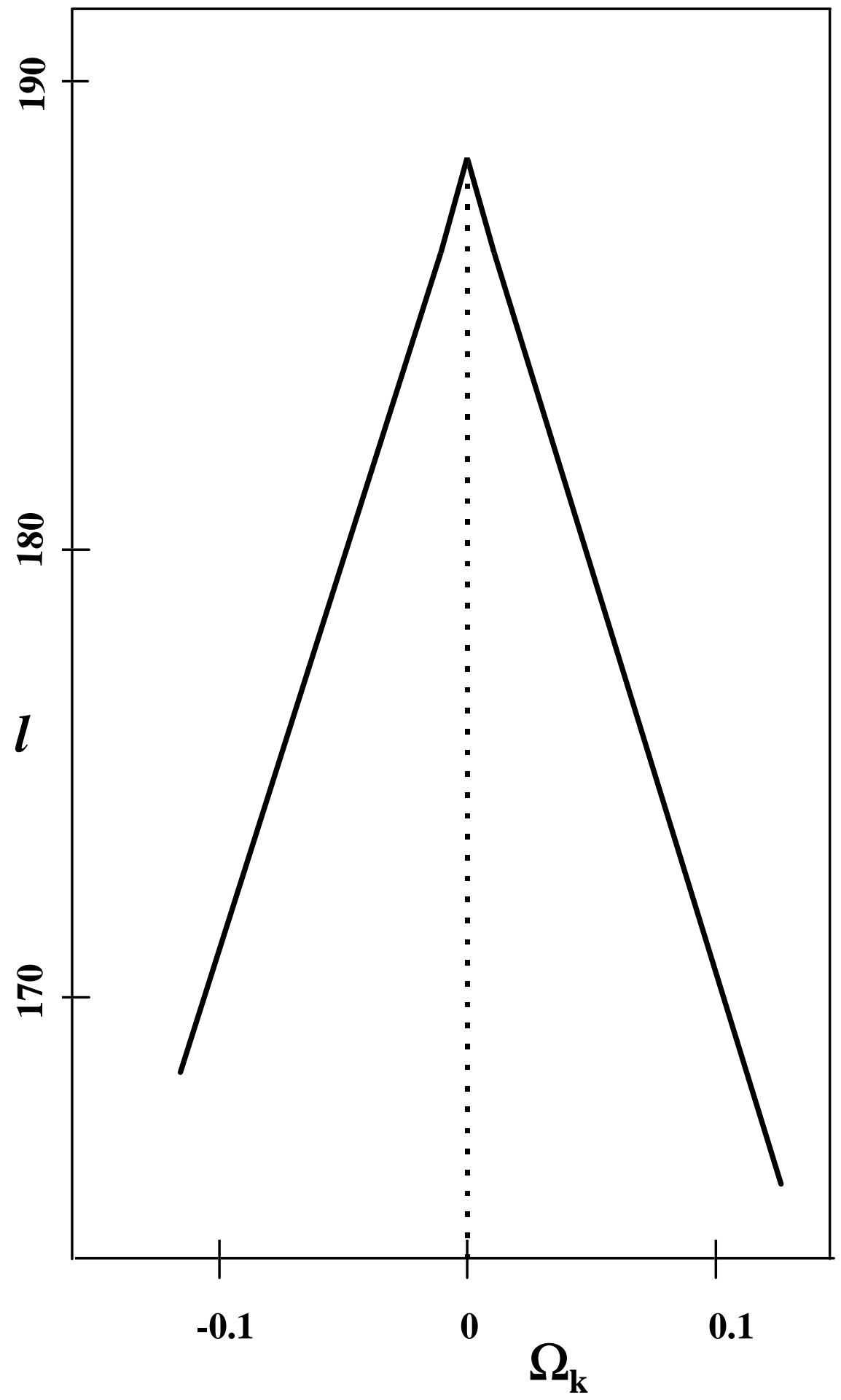

Fig. 1.- Dependence of the first Doppler peak on the curvature of the universe for a quintessence model with constant parameter $\omega=-2 / 3$. 